\title{
Windowed Fourier transform applied in the wavelength domain to process the spectral interference signals
}

\author{
P. Hlubina* , J. Luňáček, D. Ciprian, R. Chlebus \\ Department of Physics, Technical University Ostrava, 17. listopadu 15, \\ 70833 Ostrava-Poruba, Czech Republic
}

\begin{abstract}
We report on processing the spectral interference signals by a new method based on a windowed Fourier transform applied in the wavelength domain. First, the numerical simulations are performed to demonstrate high precision of the phase retrieval from the spectral signal. Second, the feasibility of the method is confirmed in processing experimental data from a dispersive Michelson interferometer comprising a cube beamsplitter made of BK7 glass. From the retrieved spectral phase difference, the effective thickness of the beamsplitter is determined precisely.
\end{abstract}

Key words: white light, spectral interferometry, dispersion, windowed Fourier transform, retrieved phase, cube beamsplitter, BK7 glass, effective thickness PACS: 06.30.Bp, 07.60.Ly, 42.25.Hz, 42.30.Rx

\section{Introduction}

White-light spectral interferometry utilizing a broadband source in combination with a standard Michelson or Mach-Zehnder interferometer has been widely used in various research areas including distance and displacement measurements [1-5], profilometry [6-9], material characterization [5,10-16] and optical communications $[17,18]$.

White-light spectral interferometry is based on the observation of spectrallyresolved interference fringes (channeled spectrum) obviously recorded far from

* Tel.: +420-597-323-134; fax: +420-597-323-139.

Email address: petr.hlubina@vsb.cz (P. Hlubina). 
a stationary phase point [19] and involves measurement of the phase or period of the spectral fringes. The channeled spectrum can be recorded in the frequency (wavenumber) or wavelength domain and can be stationary or nonstationary (without or with variations of the periodicity of the fringes). In profilometry, for example, the sample height profile can be determined by simply differentiating the spectral phase difference (spectral phase) retrieved from a single interferogram recorded in the wavenumber domain [6-9]. Similarly, the group dispersion of a sample under study placed in the interferometer can be obtained by simply differentiating the frequency-dependent spectral phase [17]. If the spectral interferogram is recorded in the wavelength domain (by the CCD array with an equal wavelength sampling), it can be transformed into the frequency domain replacing the wavelength sampling with an equal frequency sampling using suitable algorithms $[8,17,20]$. The algorithms can also be used to transform non-stationary interferograms into stationary ones. The stationary wavelength-domain channeled spectra are still appropriate to retrieve the spectral phase as a function of the wavelength $[12,18]$.

There exist a large number of algorithms suitable for the phase reconstruction from the recorded channeled spectrum. For the spectrum recorded in the frequency (wavenumber) domain, these include a five-point $[2,10]$ and a sevenpoint $[7,9]$ algorithms, a Fourier-transform algorithm [5,8] and especially for the non-stationary channeled spectrum, a wavelet-transform algorithm [15]. For the spectrum recorded in the wavelength domain, the algorithms include a two-point algorithm [11], a Fourier-transform algorithm [12,13,18], a phaselocked loop method [12] and especially for the non-stationary channeled spectrum, the Kalman filtering method [14,21]. In the latter case, the spectral phase is reconstructed from the spectral interference signal, which is obtained from the recorded channeled spectrum subtracting the effect of the reference non-channeled spectrum.

In this paper, a new method of processing the non-stationary spectral interference signals is presented, which is based on a windowed Fourier transform $[22,23]$ effectively applied in the wavelength domain. First, we performed the numerical simulations to demonstrate high precision of the phase retrieval from the spectral signal. The feasibility of the method was confirmed in processing experimental data from a dispersive Michelson interferometer to determine precisely the effective thickness of a cube beamsplitter made of BK7 glass. The spectral phase was retrieved and the effective thickness was obtained as the slope of the linear dependence of the retrieved optical path difference on the refractive index of the glass [13]. We utilized the technique to measure the effective thickness of two different cube beamsplitters. 


\section{Theoretical background}

In this section we show how a windowed Fourier transform (WFT) is defined in the spatial domain $[22,23]$ and how the spatial fringe pattern is related to the spectral interference signal. We demonstrate also the feasibility of the WFT in processing the simulated spectral interference signal, including noise, to retrieve precisely the spectral phase.

\subsection{Windowed Fourier transform}

A 1D spatial-domain fringe pattern can be generally expressed as

$$
f(x)=a(x)+b(x) \cos [\phi(x)]+n_{f}(x),
$$

where $f(x), a(x)$ and $b(x)$ are the recorded intensity, background intensity, and fringe amplitude, respectively; $\phi(x)$ is the fringe phase distribution, and $n_{f}(x)$ is the noise, which is assumed to be additive. To retrieve the phase information $\phi(x)$ from the recorded fringe intensity $f(x)$, a WFT will be used. The WFT and inverse WFT can be written as

$$
\begin{aligned}
& F(u, \xi)=\int_{-\infty}^{+\infty} f(x) g(x-u) \exp (-\mathrm{j} \xi x) \mathrm{d} x \\
& f(x)=\frac{1}{2 \pi} \int_{-\infty}^{+\infty} \int_{-\infty}^{+\infty} F(u, \xi) g(x-u) \exp (\mathrm{j} \xi x) \mathrm{d} \xi \mathrm{d} u
\end{aligned}
$$

where $F(u, \xi)$ denotes the WFT spectrum and $g(x)$ is a window, which can be chosen as a Gaussian function:

$$
g(x)=\exp \left(-x^{2} / 2 \sigma^{2}\right)
$$

where the parameter $\sigma$ controls the width of the Gaussian window. By combining Eqs. (2) and (3), we obtain

$$
f(x)=\frac{1}{2 \pi} \int_{-\infty}^{+\infty}[f(x) \otimes h(x, \xi)] \otimes h(x, \xi) \mathrm{d} \xi
$$

where $h(x, \xi)=g(x) \exp (\mathrm{j} \xi x)$ and $\otimes$ denotes a convolution, which is implemented with respect to the variable $x$. Next, an approach based on a windowed 
Fourier filtering with modifications of Eq. (5) is used [22,23]:

$$
\bar{f}(x)=\frac{1}{2 \pi} \int_{a}^{b}[\overline{f(x) \otimes h(x, \xi)}] \otimes h(x, \xi) \mathrm{d} \xi .
$$

The modifications include the thresholding denoted by the overline and the setting the integration limits from $a$ to $b$. The thresholding means that if $|f(x) \otimes h(x, \xi)|$ is smaller than a certain threshold $T$, it is treated as noise and is removed. The integration limits mean that only the desired spectrum in a limited range is selected.

After fringe pattern $\bar{f}(x)$ is synthetized, the phase $\phi(x)$ can be retrieved as

$$
\phi(x)=\operatorname{angle}[\bar{f}(x)] .
$$

The phase can be unwrapped using a simple procedure.

\subsection{Spectral interference signal}

A 1D fringe pattern in the wavelength domain, which can be recorded for example at the output of a Michelson interferometer, has the general form [4]

$$
I_{\mathrm{M}}(\lambda)=I_{\mathrm{M}}^{(0)}(\lambda)\left\{1+V_{I}(\lambda) \cos \left[(2 \pi / \lambda) \Delta_{\mathrm{M}}(\lambda)\right]\right\}
$$

where $I_{\mathrm{M}}^{(0)}(\lambda)$ is the reference spectrum, $V_{I}(\lambda)$ is the wavelength-dependent overall visibility of the spectral interference fringes and $\Delta_{\mathrm{M}}(\lambda)$ is the wavelengthdependent optical path difference (OPD) between two beams in the Michelson interferometer. The form (8) can be related to the form of the 1D spatial fringe pattern given by Eq. (1) simply replacing the spatial coordinate $x$ by the wavelength $\lambda$. Because we are retrieving only the phase, we evaluate the spectral interference signal $S_{\mathrm{M}}(\lambda)$ defined as $[14,16]$

$$
S_{\mathrm{M}}(\lambda)=I_{\mathrm{M}}(\lambda) / I_{\mathrm{M}}^{(0)}(\lambda)-1
$$

which can be represented in the form:

$$
S_{\mathrm{M}}(\lambda)=c(\lambda) \cos [\phi(\lambda)]+n_{S}(\lambda),
$$

where $S_{\mathrm{M}}(\lambda)$ and $c(\lambda)$ are the measured spectral interference signal and spectral fringe visibility, respectively; $\phi(\lambda)$ is the spectral phase to be retrieved, and $n_{S}(\lambda)$ is the noise, which is assumed to be additive. 


\subsection{Numerical simulation and processing}

In this subsection we show the feasibility of the WFT in processing the simulated spectral interference signal, including the noise, related to a specified interferometer configuration. To simulate the signal $S_{\mathrm{M}}(\lambda)$, we consider a slightly dispersive Michelson interferometer with two identical metallic mirrors and a cube beamsplitter of the effective thickness $t_{\text {ef }}$ [13]. We assume that the geometrical path lengths of the light rays in dispersive glass of the beamsplitter are not the same for both interferometer arms so that the beamsplitter can be represented by an ideal beamsplitter and a plate of the same dispersion and of the thickness $t_{\text {ef }}$ (see Fig. 1). This configuration differs substantially from that with a plate beamsplitter [24] for which a stationary phase point can be resolved in the recorded spectrum at a specific (equalization) wavelength [24]. Moreover, the dependence of the equalization wavelength on the mirror position gives either the beamsplitter group refractive index dispersion or effective thickness and no phase retrieval from the recorded spectrum is needed.

The spectral interference signal $S_{\mathrm{M}}(\lambda)$ obtained from the channelled and reference spectra, both recorded at the output of the interferometer by a fiber-optic spectrometer characterized by a Gaussian response function, can be expressed in the form of Eq. (10), in which [4]

$$
c(\lambda)=V_{I} \exp \left\{-\left(\pi^{2} / 2\right)\left[\Delta_{\mathrm{M}}^{\mathrm{g}}(\lambda) \Delta \lambda_{\mathrm{R}} / \lambda^{2}\right]^{2}\right\}
$$

and

$$
\left.\phi(\lambda)=(2 \pi / \lambda) \Delta_{\mathrm{M}}(\lambda)\right]
$$

In Eq. (11), $V_{I}$ is a visibility term and $\Delta \lambda_{\mathrm{R}}$ denotes the width of the spectrometer response function. The wavelength-dependent $\mathrm{OPD} \Delta_{\mathrm{M}}(\lambda)$ is given by

$$
\Delta_{\mathrm{M}}(\lambda)=2 L+2 n(\lambda) t_{\mathrm{ef}}
$$

where $2 L$ is the difference of path lengths between the interfering beams in the air whose dispersion is neglected and $n(\lambda)$ is the wavelength-dependent refractive index of the beamsplitter material. In Eq. (11), $\Delta_{\mathrm{M}}^{\mathrm{g}}(\lambda)$ is the group OPD corresponding to the OPD $\Delta_{\mathrm{M}}(\lambda)$ and is given by

$$
\Delta_{\mathrm{M}}^{\mathrm{g}}(\lambda)=2 L+2 N(\lambda) t_{\mathrm{ef}},
$$


where $N(\lambda)$ is the wavelength-dependent group refractive index given by:

$$
N(\lambda)=n(\lambda)-\lambda \frac{\mathrm{d} n(\lambda)}{\mathrm{d} \lambda}
$$

To show the feasibility of the WFT in processing the simulated spectral interference signal, first we choose the reference spectrum $I_{\mathrm{M}}^{(0)}(\lambda)$ (known from a series of measurements and affected by the noise) and simulated the channeled spectrum $I_{\mathrm{M}}(\lambda)$ calculated for 2048 wavelengths with an equal sampling in the range from 350 to $1000 \mathrm{~nm}$. Fig. 2 shows the reference spectrum by the dashed line and the channeled spectrum by the solid line in a narrower wavelength range from 450 to $900 \mathrm{~nm}$. The channeled spectrum $I_{\mathrm{M}}(\lambda)$ in Eq. (9) was calculated from the simulated spectral interference signal $S_{\mathrm{M}}(\lambda)$ given by Eq. (10). The signal is shown in Fig. 3 by the solid line when Eqs. (10) to (15) were used and when we considered the beamsplitter made of pure BK7 optical glass with the effective thickness $t_{\mathrm{ef}}=-10 \mu \mathrm{m}$, the width of the spectrometer response function $\Delta \lambda_{\mathrm{R}}=3 \mathrm{~nm}$, the visibility term $V_{I}=0.9$ and the difference of path lengths between the interfering beams in the air $2 L=54.6 \mu \mathrm{m}$. In the spectral signal, whose period is longer for longer wavelengths, the additive noise with zero mean value was included.

Second, the simulated spectral signal was processed by the WFT, parameters of which were optimized. The processing was performed for the signal including all 2048 wavelength samples with the window width $\sigma$ and the threshold $T$ that were modified. In the processing of the signal $S_{\mathrm{M}}(\lambda)$ we used the wavelengthdomain representation of the WFT with a simple algorithm presented in [23] to retrieve the unwrapped phase function $\Phi(\lambda)$, which is known with the ambiguity of $m 2 \pi$, where $m$ is an integer. Using the algorithm we retrieved the phase function $\phi(\lambda)$ in the range from 450 to $900 \mathrm{~nm}$. The precision of the phase retrieval by the WFT is demonstrated by the phase error distribution. It should be stressed here that this procedure differs substantially from that presented in [16], where no optimization of the parameters was performed and an additional smoothing algorithm was used to retrieve the phase.

We started with the parameters $\sigma=20$ and $T=0$ and Fig. 4 shows by the solid line the corresponding phase error distribution with larger errors for the wavelengths longer than $600 \mathrm{~nm}$. The apparent oscillatory behavior is resolved which can be attributed to too narrow window to suppress the effect of the non-stationarity of the signal (longer period for the longer wavelengths). The effect of the wider window is demonstrated in Fig. 4 by the dashed line which shows the phase error distribution corresponding to the parameters $\sigma=50$ and $T=0$. The effect of the additive noise in the spectral signal can be suppressed by non-zero threshold as is demonstrated in Fig. 4 by the bold line which shows the phase error distribution corresponding to the parameters $\sigma=50$ and $T=0.5$. In Fig. 3 is then shown by the dashed line a signal $\cos [\phi(\lambda)]$ demonstrating 
good phase reconstruction of the original signal.

If one needs to retrieve the spectral phase from more non-stationary signals, two different approaches can be proposed. First one can utilize dividing the spectral signal into at least two parts in different and adjacent wavelength regions in which the signal is locally more stationary and in which the above procedure can be applied with different and appropriate window widths. Second one can utilize a multiscale WFT [26] effectively applied in the wavelength domain.

\section{Experimental configuration}

The experimental setup used in the application of spectral-domain white-light interferometry to record channeled spectra and to process them by the WFT is shown in Fig. 1. From the retrieved spectral phase, the effective thickness of a cube beamsplitter, which is an important parameter for interferometric applications with a Michelson interferometer $[14,16]$, is determined. The setup consists of a white-light source, a halogen lamp with launching optics, an optical fiber and a collimating lens, a bulk-optic Michelson interferometer with a cube beamsplitter, a micropositioner connected to one of the mirrors, a microscope objective, micropositioners, a read optical fiber, a miniature fiber-optic spectrometer S2000, an A/D converter and a personal computer. The beamsplitter is made of BK7 glass. The resolution of the fiber-optic spectrometer S2000 (Ocean Optics, Inc.), the design of which was reported previously [14], is in our case given by the effective width of the light beam from a core of the read optical fiber: we used the read optical fiber of a $50 \mu \mathrm{m}$ core diameter to which a Gaussian response function corresponds. The spectral interferograms were recorded at room temperature in the rigid positions of the interferometer with known positions of mirror 2 . The measurements of the effective thickness were performed for two different beamsplitters.

\section{Experimental results and discussion}

The effective thickness $t_{\text {ef }}$ of the first cube beamsplitter made of BK7 glass was measured in two steps. First, the channelled spectrum $I_{\mathrm{M}}(\lambda)$ was recorded for a suitable OPD adjusted in the Michelson interferometer. Second, the reference spectral intensity $I_{\mathrm{M}}^{(0)}(\lambda)$ was recorded for such an OPD so that channeled spectrum was smoothed due to the limited resolving power of the spectrometer. The two recorded spectra were used to determine the spectral interference signal (9) which is shown in Fig. 5 by the solid line. By processing the spectral signal using the WFT with the parameters $\sigma=50$ and $T=0.5$, the 
unwrapped phase function $\Phi(\lambda)$ was determined. Fig. 5 shows by the dashed line a signal $\cos [\Phi(\lambda)]$ and demonstrates good phase reconstruction of the original signal. The phase function $\Phi(\lambda)$ is known with the ambiguity of $m 2 \pi$, where $m$ is an integer. Using Eqs. (12) and (13), the relation for the OPD $\Delta_{\mathrm{M}}(\lambda)$ between interfering beams

$$
\Delta_{\mathrm{M}}(\lambda)=[\Phi(\lambda) /(2 \pi)+m] \lambda=2 L+2 n(\lambda) t_{\mathrm{ef}}
$$

has to be fulfilled. It results from Eq. (16) that knowing the unwrapped phase function $\Phi(\lambda)$ and the dispersion of the refractive index $n(\lambda)$, the interference order $m$ and thus the absolute spectral phase $\phi(\lambda)$ and OPD $\Delta_{\mathrm{M}}(\lambda)$ can be determined [13]. The interference order $m$ of such a value has to be chosen so that the OPD $\Delta_{\mathrm{M}}(\lambda)$ between beams in the interferometer is linearly dependent on the refractive index $n(\lambda)$. Two parameters (the shift and slope) of the corresponding linear function give the difference $2 L$ and the twofold effective thickness $2 t_{\text {ef }}$. The procedure has already been used in the absolute phase retrieval from the recorded channeled spectra that included the effect of thin films $[14,16]$.

The above procedure is illustrated in Fig. 6 , in which the OPD $\Delta_{\mathrm{M}}(\lambda)$ between beams in the interferometer given by Eq. (16) is evaluated for such a choice of the interference order $m$ so that the dependence of the OPD $\Delta_{\mathrm{M}}(\lambda)$ on the refractive index $n(\lambda)$ of BK7 glass at room temperature [25] deviates minimally from linear dependence. Fig. 6 shows the dependence by the solid line together with the corresponding linear fit characterized by parameters that give the beamsplitter effective thickness $t_{\mathrm{ef}}=45.399 \mu \mathrm{m}$ with a standard deviation of $0.008 \mu \mathrm{m}$ and the position of the interferometer mirror $L=-60.009 \mu \mathrm{m}$ with a standard deviation of $0.012 \mu \mathrm{m}$. The results obtained demonstrate that the interferometer with the first cube beamsplitter is slightly dispersive [14] so that the stationary phase point [19] cannot be resolved in the recorded spectra.

The effective thickness $t_{\text {ef }}$ of the second cube beamsplitter made of BK7 glass was measured even if the stationary phase point was resolved in some of the recorded spectra. To apply the above-presented method for measuring the effective thickness, we adjusted such an OPD in the interferometer to be far from the stationary phase point and to resolve the channeled spectrum. Fig. 7 shows by the solid line the spectral interference signal (9) obtained from two recorded spectra, one of them was the reference spectrum. By processing the spectral signal using the WFT with the parameters $\sigma=50$ and $T=0.5$, the unwrapped phase function $\Phi(\lambda)$ was determined. Fig.7 shows by the dashed line a signal $\cos [\Phi(\lambda)]$ and demonstrates good phase reconstruction of the original signal. 
Knowing both the unwrapped phase function $\Phi(\lambda)$ and the refractive index dispersion $n(\lambda)$ of the beamsplitter made of BK7 glass, the unknown interference order $m$ in Eq. (16) and thus the absolute OPD $\Delta_{\mathrm{M}}(\lambda)$ between beams in the interferometer were determined. The procedure used is illustrated in Fig. 8, in which the OPD $\Delta_{\mathrm{M}}(\lambda)$ given by Eq. (16) is evaluated from the known unwrapped phase $\Phi(\lambda)$ and such a choice of the interference order $m$ so that the dependence of the OPD $\Delta_{\mathrm{M}}(\lambda)$ on the refractive index $n(\lambda)$ of BK7 glass deviates minimally from linear dependence. Fig. 8 shows the dependence by the solid line together with the corresponding linear fit characterized by parameters that give the effective thickness $t_{\mathrm{ef}}=-126.066 \mu \mathrm{m}$ with a standard deviation of $0.005 \mu \mathrm{m}$ and the position of the interferometer mirror $L=202.439 \mu \mathrm{m}$ with a standard deviation of $0.007 \mu \mathrm{m}$. The negative effective thickness of the beamsplitter means that the corresponding dispersive plate is in the other arm of the interferometer than is depicted in Fig. 1.

\section{Conclusions}

A new method of processing the spectral interference signals based on the WFT effectively applied in the wavelength domain has been presented. The numerical simulations have been performed to demonstrate high precision of the phase retrieval from the spectral signal. Phase error distributions corresponding to different parameters have also been presented. The feasibility of the method, which is easy to implement, has been confirmed in processing experimental data from a dispersive Michelson interferometer to determine precisely the effective thickness of a cube beamsplitter made of BK7 glass. The spectral phase was retrieved to obtain the effective thickness as the slope of the linear dependence of the retrieved OPD on the refractive index of the glass. The effective thickness of two different cube beamsplitters was measured by the technique. The results obtained are important from the point of view of processing the channeled spectra. These can be the results of implementation of white-light spectral interferometry in various research fields, including distance and displacement measurements, profilometry, material characterization and optical communications.

\section{Acknowledgements}

The research was partially supported by the Grant Agency of the Czech Republic (projects 102/06/0284, 202/06/0531) and by the grant MSM6198910016. 


\section{References}

[1] L.M. Smith, C.C. Dobson, Appl. Opt. 28 (1989) 3339.

[2] U. Schnell, E. Zimmermann, R. Dändliker, Pure Apl. Opt. 4 (1995) 643.

[3] P. Hlubina, Opt. Commun. 212 (2002) 65.

[4] P. Hlubina, J. Mod. Opt. 51 (2004) 537.

[5] K.-N. Joo, S.-W. Kim, Opt. Express 14 (2006) 5954.

[6] J. Schwider, L. Zhou, Opt. Lett. 19 (1994) 995.

[7] P. Sandoz, G. Tribillon, H. Perrin, J. Mod. Opt. 43 (1996) 701.

[8] T. Endo, Y. Yasuno, S. Makita, M. Itoh, T. Yatagai, Opt. Express 13 (2005) 695.

[9] D. Reolon, M. Jacquot, I. Verrier, G. Brun, C. Veillas, Opt. Express 13 (2005) 128.

[10] U. Schnell, R. Dändliker, S. Gray, Opt. Lett. 21 (1996) 528.

[11] Y. Liang, C.P. Grover, Appl. Opt. 37 (1998) 4105.

[12] I. Gurov, P. Hlubina, V. Chugunov, Meas. Sci. Technol. 14 (2003) 122.

[13] P. Hlubina, Acta Phys. Slov. 55 (2005) 387.

[14] P. Hlubina, D. Ciprian, J. Luňáček, M. Lesňák, Opt. Express 14 (2006) 7678.

[15] D. Reolon, M. Jacquot, I. Verrier, G. Brun, C. Veillas, Opt. Express 14 (2006) 12744 .

[16] P. Hlubina, D. Ciprian, J. Luňáček, R. Chlebus, Appl. Phys. B 88 (2007) 397.

[17] J.Y. Lee, D.Y. Kim, Opt. Express 14 (2006) 11608.

[18] Y.-P. Li, C.-C. Lee, Opt. Commun. 265 (2006) 406.

[19] C. Sáinz, P. Jourdain, R. Escalona, J. Calatroni, Opt. Commun. 110 (1994) 381.

[20] C. Dorrer, N. Belabas, J.-P. Likforman, M. Joffre, J. Opt. Soc. Amer. B 17 (2000) 1795.

[21] I. Gurov, E. Ermolaeva, A. Zakharov, J. Opt. Soc. Amer. A 21 (2004) 242.

[22] K. Qian, Appl. Opt. 43 (2004) 2695.

[23] K. Qian, Opt. Lasers Eng. 45 (2007) 304.

[24] P. Hlubina, Opt. Commun. 193 (2001) 1.

[25] Schott Computer Glass Catalog 1.0, Schott Glasswerke, Mainz, Germany, 1992.

[26] J. Zhong, H. Zeng, Appl. Opt. 46 (2007) 2670. 


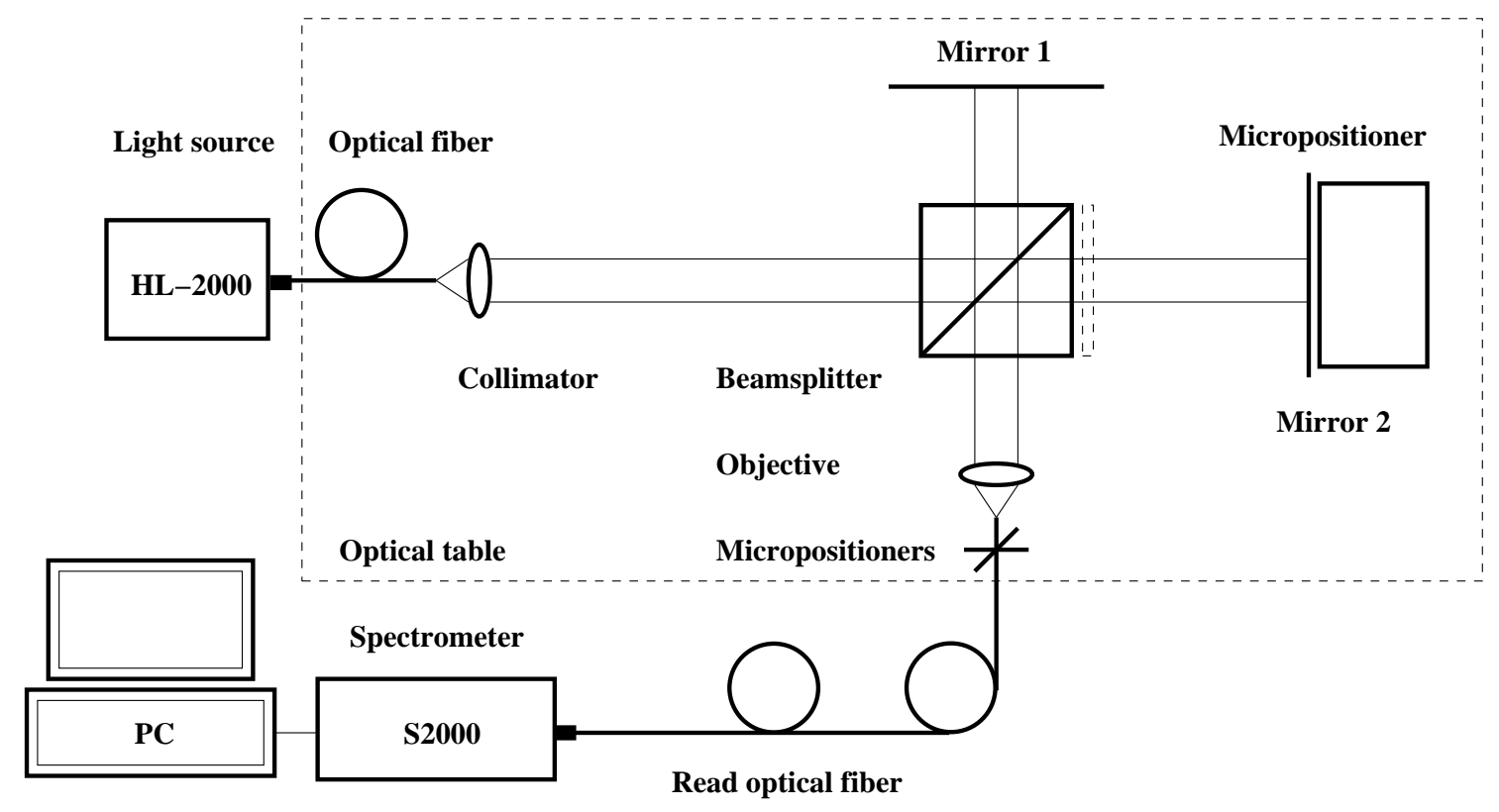

Fig. 1. Experimental setup with a Michelson interferometer to measure the spectral interference signals.

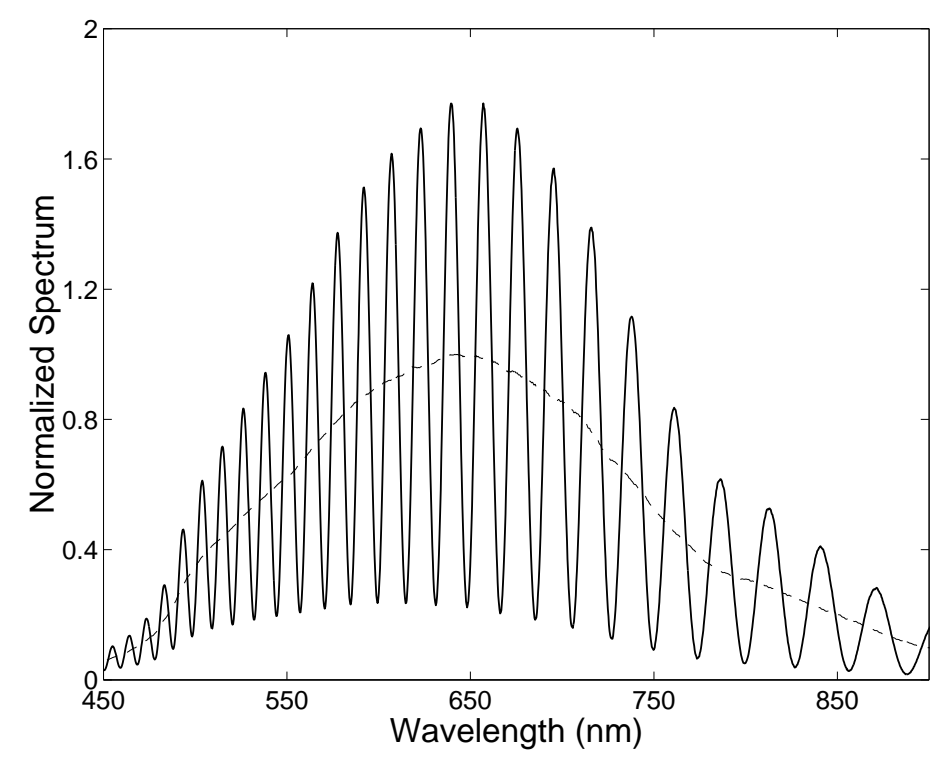

Fig. 2. Theoretical channeled spectrum (solid) and reference spectrum (dashed). 


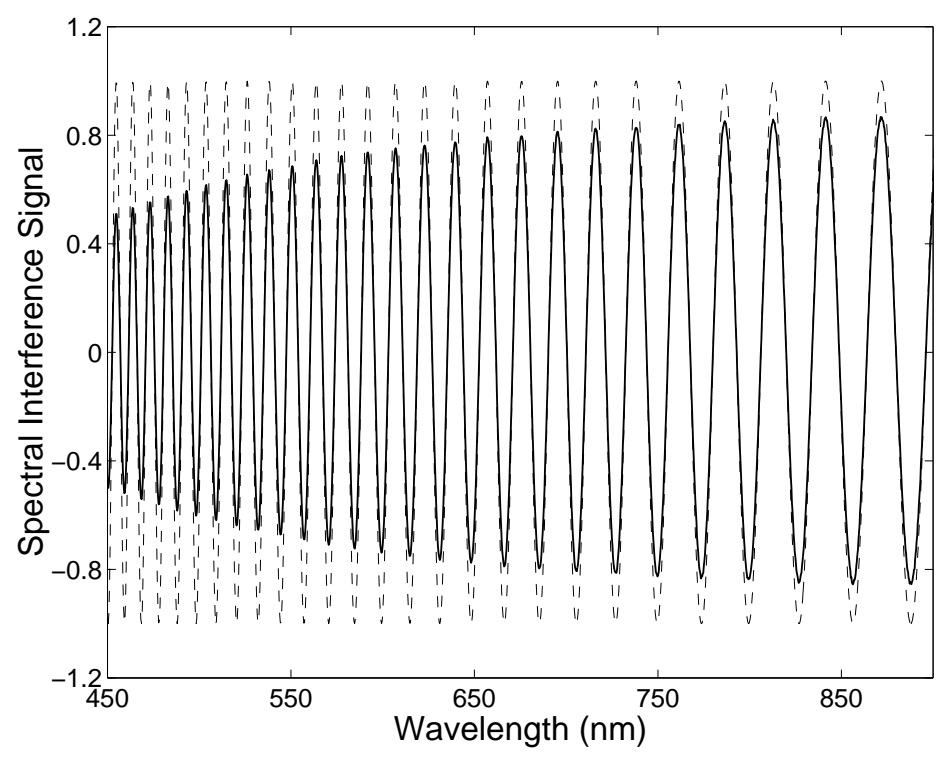

Fig. 3. Comparison of the theoretical spectral interference signal with the signal (dashed) that uses the retrieved phase function.

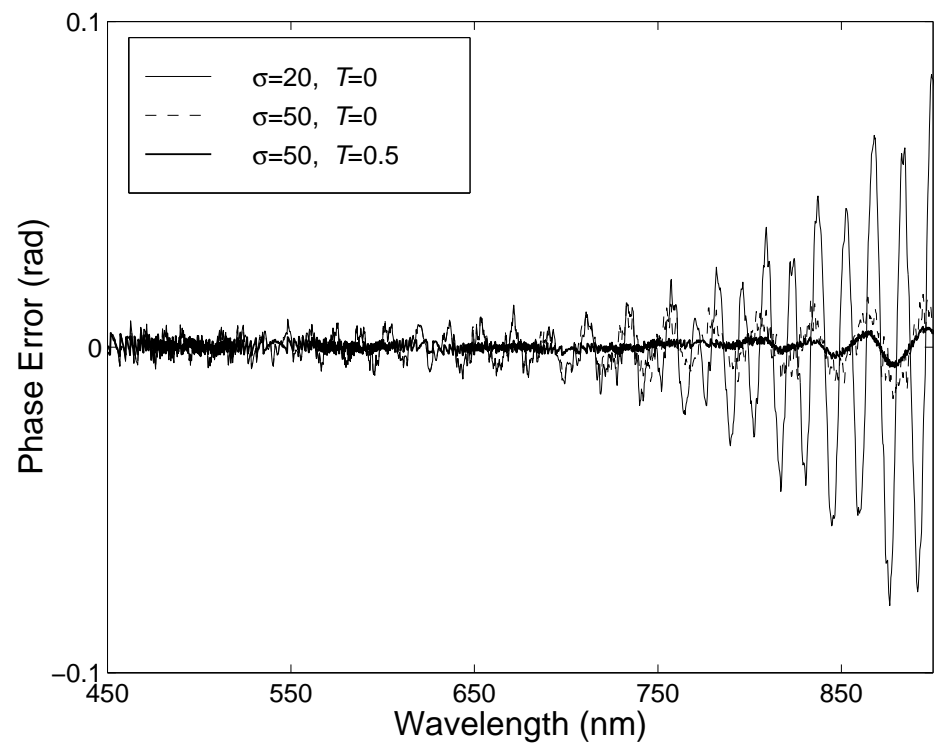

Fig. 4. Error distributions of the phase retrieved by use of the WFT characterized by different parameters: the window width $\sigma$ and the threshold $T$. 


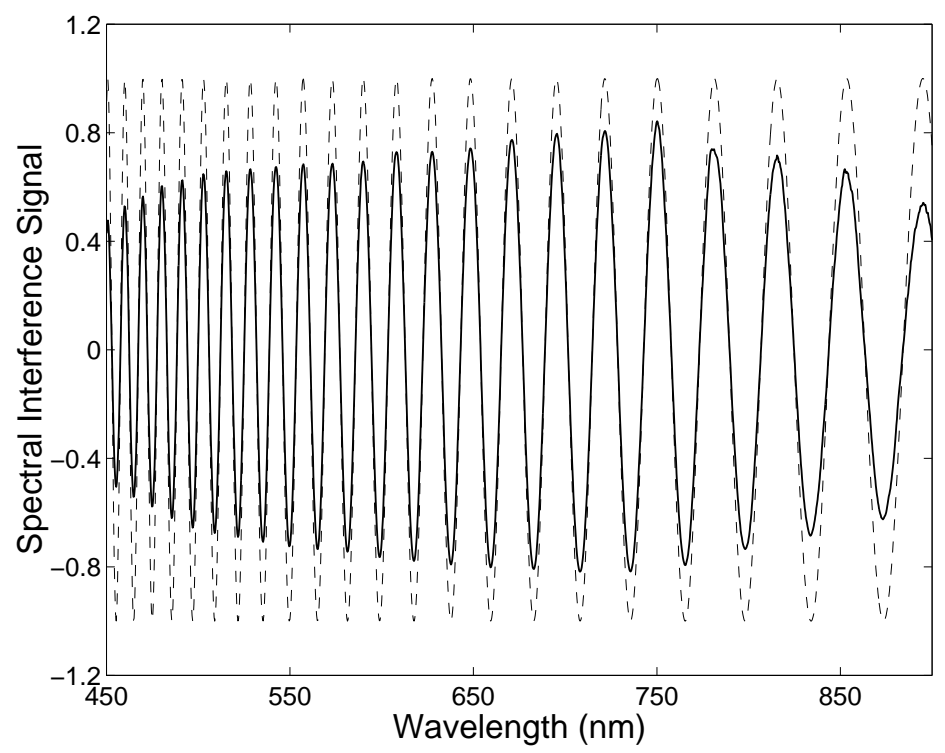

Fig. 5. Comparison of the measured spectral interference signal (solid) with the signal (dashed) that uses the retrieved phase function.

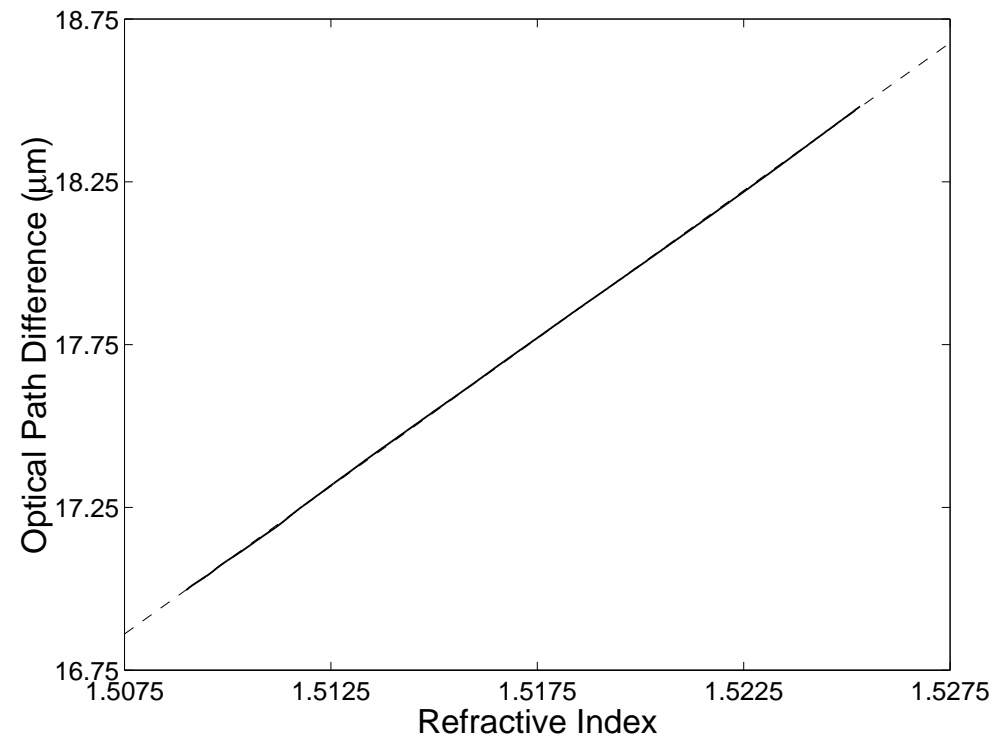

Fig. 6. The OPD between interfering beams as a function of the refractive index of BK7 glass (solid) together with a linear fit (dashed). 


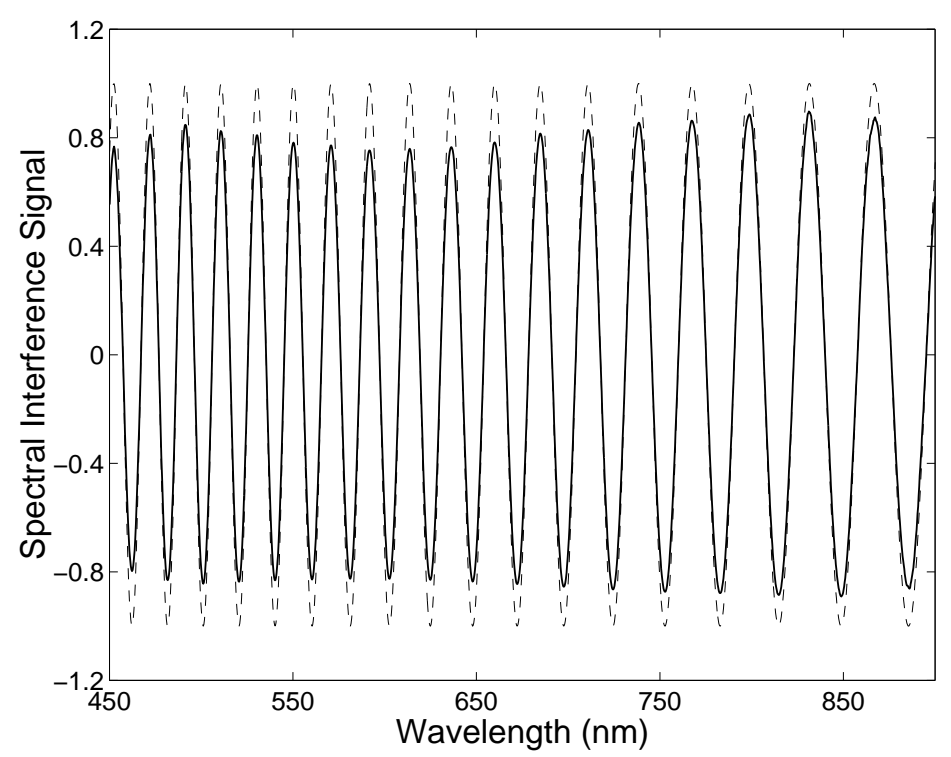

Fig. 7. Comparison of the measured spectral interference signal (solid) with the signal (dashed) that uses the retrieved phase function.

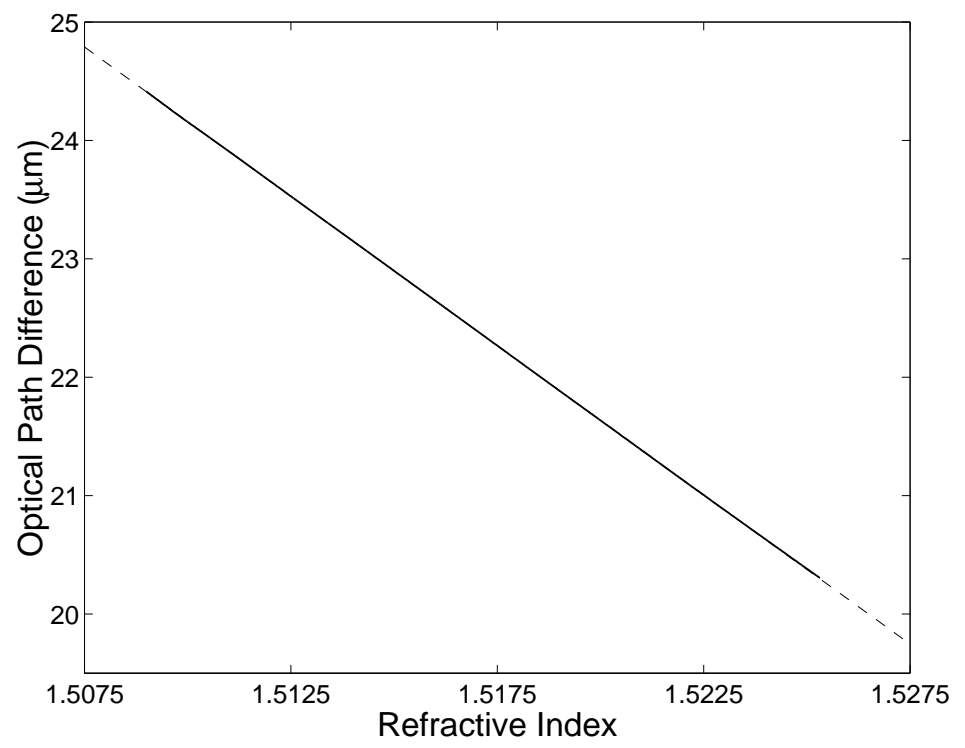

Fig. 8. The OPD between interfering beams as a function of the refractive index of BK7 glass (solid) together with a linear fit (dashed). 